\title{
Amyloid- $\beta$ peptide-induced extracellular S100A9 depletion is associated with decrease of antimicrobial peptide activity in human THP-1 monocytes
}

Eun Ok Lee', Ji Hye Yang ${ }^{1}$, Keun-A Chang ${ }^{3}$, Yoo-Hun Suh ${ }^{2}$ and Young Hae Chong ${ }^{1 *}$

\begin{abstract}
Background: S100A9 protein (myeloid-related protein MRP14, also referred to as calgranulin B) is a reliable marker of inflammation, an important proinflammatory factor of innate immunity and acts as an additional antimicrobial peptide in the innate immune system. Evidence indicates that S100A9 contributes to Alzheimer's disease (AD) pathology, although the precise mechanisms are not clear.
\end{abstract}

Methods: We were interested to study the mechanisms of S100A9 release upon A 1 1-42 stimulation, the potential roles of extracellular S100A9 depletion in Aß-induced cytotoxicity, and the interaction with innate immune response in THP-1 monocytic cells that have been challenged with mostly Aß1-42 monomers instead of oligomers. We used protein preparation, $\mathrm{Ca}^{2+}$ influx fluorescence imaging, MTT assay, siRNA knockdown, colony forming units (CFUs) assay and western blotting techniques to perform our study.

Results: A 1-42 monomers elicited a marked decrease of S100A9 release into the cell culture supernatant in a dose-dependent manner in human THP-1 monocytes. This reduction of S100A9 release was accompanied by an increase of intracellular $\mathrm{Ca}^{2+}$ level. AB1-42-mediated decrease of S100A9 release was not associated with AB1-42induced cytotoxicity as measured by MTT reduction assay. This observation was confirmed with the recombinant S100A9, which had little effect on AB1-42-induced cytotoxicity. Moreover, depletion of S100A9 with siRNA did not significantly evoke the cell toxicity. On the other hand, AB1-42-induced extracellular S100A9 depletion resulted in decreased antimicrobial activity of the culture supernatant after A 1-42 stimulation. Immunodepletion of S100A9 with anti-S100A9 also decreased the antimicrobial peptide activity of the vehicle treated culture supernatant. Consistently, the recombinant S100A9 clearly elicited the antimicrobial peptide activity in vitro, confirming the observed antimicrobial activity of S100A9 in the culture supernatant.

Conclusion: Collectively, our findings suggest that the mostly monomeric form of A $31-42$ negatively regulates the innate immune system by down-regulating the secretion of S100A9, which is likely a main mediator of antimicrobial activity in the conditioned media of human THP-1 monocytes.

Keywords: Alzheimer's disease, A 1-42, cytotoxicity, S100A9, Antimicrobial activity, Innate immune response

\footnotetext{
* Correspondence: younghae@ewha.ac.kr

'Department of Microbiology, School of Medicine, Ewha Medical Research Institute, Ewha Womans University, 911-1, Mok-6-dong, Yangcheonku, Seoul 158-710, Republic of Korea

Full list of author information is available at the end of the article
} 


\section{Background}

Alzheimer's disease (AD) is the most common and still incurable form of dementia, which primarily affects the population over the age of 60 years. Amyloid beta $(A \beta)$ deposition, neurofibrillary tangle formation and neuroinflammation are the major pathogenetic mechanisms that, in concert, lead to neocortical and hippocampal atrophy, memory dysfunction and decline of cognition in $\mathrm{AD}[1,2]$. There are currently no curative or effective clinical treatments for AD [3].

The innate immune response and inflammatory signaling play determinant roles in brain homeostasis, neuroprotection and repair. However, altered or excessive signaling in these injury defense systems contributes to neuroinflammation and the irreversible degeneration of brain cells [4]. Extensive innate immune gene activation reflecting chronic innate immune activation could accompany brain aging, increasing vulnerability to $\operatorname{cog}$ nitive decline and neurodegeneration, consistent with the emerging idea of a critical involvement of inflammation in the earliest stages of $\mathrm{AD}$ [5]. Thus, clinical pharmaceutical trials aimed at modulating the immune system in $\mathrm{AD}$ have largely focused on dampening down central proinflammatory innate immunity and the manipulation of systemic immunity, and its communication with the central nervous system (CNS) [6].

Calgranulins reflecting calcium-binding properties and high expression in granulocytes are comprised of three proteins: S100A8 (calgranulin A, also termed as MRP8), S100A9 (calgranulin B, also termed as MRP14) and S100A12 (Calgranulin C). They are predominantly expressed by neutrophils, monocytes and activated macrophages in inflamed tissue [7]. These S100 calciumbinding proteins are important molecular mediators in a range of diseases, including microbial infections. In particular, S100A9 protein is a reliable marker of inflammation and an important proinflammatory factor of innate immunity. Elevated plasma levels of S100A9 are associated with inflammatory disorders such as chronic bronchitis, cystic fibrosis and rheumatoid arthritis [8].

The extracellular roles of S100A9 in leukocyte migration and chemotaxis, leukocyte activation, oxidant scavenging, and their relevance in inflammatory processes are in particular implicated [7,9,10]. Recent reports have also suggested that S100A9 acts as an additional antimicrobial peptide in the innate immune system, which provides immediate protection for the host against microbial challenge by recognizing the presence of microorganisms and preventing their tissue invasion, thus limiting microbial proliferation and inflammation $[11,12]$.

Altered expression/function of these S100 protein members [13] has been associated with neurological diseases such as cerebral ischemia [14] and traumatic brain injury [15]. Earlier studies demonstrated that S100 proteins assemble within neuritic plaques and reactive glia, which may serve to prolong neuroinflammation associated with the pathogenesis of AD [16,17]. Our recent study showed that S100A9 expression was increased in the brains of $\mathrm{Tg} 2576$ mice, as well as in AD brains, which proposed its potential role in the neuroinflammation related to the pathogenesis of $\mathrm{AD}[18,19]$. Another recent study reported that S100A9 interacts with $A \beta$ and induces fibrillization, further supporting its association with $\mathrm{AD}$ [20]. However, a mechanistic link between S100A9 and AD pathology, and the detailed molecular mechanism have not been clearly shown.

We focused our research on the mechanisms of S100A9 release upon stimulation with mostly A $\beta 1-42$ monomers, the potential roles of extracellular S100A9 depletion in $\mathrm{A} \beta$-induced cytotoxicity, and the interaction with innate immune response in THP-1 monocytic cells that have been challenged with $A \beta 1-42$ monomers instead of oligomers. The results of the present study show that the mostly monomeric form of $A \beta 1-42$ negatively regulates the innate immune system by down-regulating the release of S100A9, which is likely a main mediator for the antimicrobial action in the culture media of human THP-1 monocytes.

\section{Materials and methods \\ Materials}

Synthetic siRNA for S100A9 and the non-specific control pool were purchased from Santa Cruz Biotechnology (Santa Cruz, CA, USA). Lipofectamine 2000 was purchased from Invitrogen (Carlsbad, CA, USA). AntiS100A9 was acquired from R\&D Systems (Minneapolis, MN, USA). Horseradish peroxidase-conjugated antimouse IgG and anti-rabbit IgG were obtained from Jackson ImmunoResearch (West Grove, PA, USA). Actinomycin, inhibitor of de novo mRNA expression, and cycloheximide, inhibitor of protein synthesis, were obtained from Calbiochem (La Jolla, CA, USA). The 3 (4,5-dimethylthiazol-2-yl)-2,5-diphenyltetrazolium bromide (MTT) was obtained from United States Biochemical (Cleveland, OH, USA). The $\mathrm{Ca}^{2+}$ ionophore, ionomycin, and an endoplasmic reticulum $\mathrm{Ca}^{2+}$ pump inhibitor, thapsigargin, were acquired from Sigma-Aldrich (St Louis, MO, USA). Anti- $\beta$-actin antibody and other chemicals, including 1,2-bis(o-aminophenoxy)ethane-N, $\mathrm{N}, \mathrm{N}^{\prime}, \mathrm{N}^{\prime}$-tetraacetic acid (BAPTA) and ethylene glycol tetraacetic acid (EGTA), were also acquired from SigmaAldrich.

\section{Preparation of $A \beta$ peptides}

A $\beta 1-42$ peptide was purchased from American Peptide Company (Sunnyvale, CA, USA) and prepared before use as previously described [21]. A $31-42$ peptide was 
dissolved at $5 \mathrm{mM}$ in dimethyl sulfoxide and diluted at $250 \mu \mathrm{M}$ in double-distilled water before experiments. This preparation contains the mostly monomeric form of $A \beta 1-42$ and very small amounts of dimers with larger oligomers up to 6-mers [21].

\section{Preparation of recombinant S100A9 protein}

Human recombinant (r) S100A9 was obtained from Dr Tessier at Laval University Hospital Center (SainteFoy, Québec, Canada), expressed in Escherichia coli and purified by previously defined protocols [22]. The purity of protein was verified by sodium dodecyl sulfate polyacrylamide gel electrophoresis (SDS-PAGE). Specificity of S100A9-mediated effect was controlled by THP-1 cell treatment with heat-inactivated rS100A9 (rS100A9 $\mathrm{hi}$ ) prepared by incubation at $85^{\circ} \mathrm{C}$ for 2 hours.

\section{Cell culture}

The human monocytic cell line THP-1 was obtained from ATCC (Rockville, MD, USA) and maintained in RPMI-1640 containing 10\% heat-inactivated fetal calf serum as previously described [21]. THP-1, a mononuclear cell line of human origin, has been widely used as a model of human monocytes/macrophages or microglia not only because of its functional and morphological similarities, including its capacity to activate signal transduction pathways, but also because of functional differences in the metabolism of rodent and human microglial cells as previously described [23].

\section{Experimental treatment}

After being washed, THP-1 cells were incubated with serum-free RPMI-1640 supplemented with $0.5 \%$ glucose for 1 hour at $37^{\circ} \mathrm{C}$ before stimulation. The cells were then stimulated by the addition of the mostly monomeric form of A $1-42$ peptide for 24 hours in the presence or absence of rS100A9 or $\mathrm{rS100A} 9_{\mathrm{hi}}$. In some experiments, cells were incubated with ionomycin or thapsigargin to determine the effect of increase of intracellular $\mathrm{Ca}^{2+}$ level. To deplete extracellular or intracellular $\mathrm{Ca}^{2+}$, cells were pretreated for 1 hour with ethylene glycol tetraacetic acid (EGTA) or BAPTA, and further incubated for 24 hours in the presence or absence of A $\beta 1-42$ monomers. All concentrations were selected on the basis of the maximal effects of the drugs on their specified targets. Vehicles were treated identically, but did not contain $A \beta 1-42$ or pharmacological agents as described above. Vehicle alone exerted no detectable effects on cell viability. After stimulation with A $\beta 1-42$ and/or the specific agents for 24 hours, total cell lysate and the supernatant were prepared and stored at $-20^{\circ} \mathrm{C}$ until use for quantification of S100A9 release by western blot analysis. The supernatant was also analyzed in parallel for antimicrobial activities.

\section{MTT assay}

The viability of cells was analyzed by the MTT assay to assess mitochondrial dehydrogenase activity as previously described [24]. Only viable cells are able to reduce MTT into a formazan product by mitochondrial dehydrogenase. After 24 hours of treatment of THP-1 cells with A $31-42$ and/or rS100A9, MTT was added to the medium $(1 \mathrm{mg} / \mathrm{ml})$ and incubated for 4 hours at $37^{\circ} \mathrm{C}$. The medium was removed and the cells were diluted in $120 \mu \mathrm{l}$ of $1 \mathrm{~N} \mathrm{HCl}$ :isopropyl alcohol (1:24) and incubated for 30 minutes at room temperature with shaking. The relative formazan concentration of each supernatant was measured by determination of the absorbance at $570 \mathrm{~nm}$ in a microplate reader.

\section{Calcium imaging and fluorescence measurements}

To visualize intracellular steady-state $\mathrm{Ca}^{2+}$ levels, THP-1 cells were stained by adding Fluo $3 \mathrm{~A}$ in its acetoxymethyl ester form (Fluo-3 AM) to $5 \mu \mathrm{g} / \mathrm{ml}$ culture media throughout $A \beta 1-42$ or vehicle treatment as previously described [18]. $\mathrm{Ca}^{2+}$ influx fluorescence images were captured after treatment as indicated. Images were recorded using an Axiovert 200 inverted microscope and analyzed with the KS 300 analysis program (Zeiss, Oberkochen, Germany). An increase in intracellular $\mathrm{Ca}^{2+}$ level in the different cultures was expressed as fold of the response of the vehicle treated controls for each individual experiment.

\section{siRNA studies}

Synthetic siRNA for S100A9 and the non-specific control pool were purchased from Santa Cruz Biotechnology, and transfection of the RNA oligonucleotide was performed using Lipofectamine 2000. THP-1 cells were treated with Lipofectamine 2000 (mock transfection), siRNA or non-specific RNA pool at the concentrations indicated. After 24 hours of transfection, the cell viability was measured by the MTT method.

\section{E coli culture and treatment}

E coli strain LE392 was used throughout this study. Colonies from agar were transferred by sterile loop to growth media and incubated aerobically in Luria Broth (Conda, Madrid, Spain) for 2 hours at $37^{\circ} \mathrm{C}$, to generate mid-logarithmic growth cultures for use as inoculates in experiments. Bacteria inoculum cell densities were normalized to $5 \times 10^{5}$ cells $/ \mathrm{ml}$ immediately before use. After stimulation with A $\beta 1-42$, the supernatant collected was mixed with $E$ coli in the ratio of $1: 1$. rS100A9 and rS100A9 $_{\text {hi }}$ were directly diluted into serum-free RPMI-1640 and also mixed with E coli. All stocks were incubated for 2 hours at $37^{\circ} \mathrm{C}$. In some experiments, rS100A9 was preincubated with antiS100A9 antibodies for 2 hours at $37^{\circ} \mathrm{C}$ before use. 
Experiments included control serial dilutions of medium or buffer vehicle alone.

\section{Colony forming unit (CFU) assay}

Serial dilutions of incubants were prepared and streaked onto the surface of Luria broth agar (Miller's LB agar). The agar plates were then incubated overnight at $37^{\circ} \mathrm{C}$ and CFUs counted as previously described [25].

\section{Preparation of human peripheral blood mononuclear cells (PBMC)}

Human PBMC were isolated from peripheral blood of healthy subjects as previously described [24] and used as a positive control for S100A9 in western blot analysis. Preparations contained approximately $10 \%$ monocytes, $90 \%$ lymphocytes and $<1.5 \%$ granulocytes.

\section{Electrophoresis and western blotting}

Immunoblotting was conducted as previously described $[24,26]$. Equal quantities of sample proteins were separated on the basis of molecular weight by $10 \%$ SDS-PAGE and transferred to polyvinylidene difluoride membranes (Millipore, Bedford, MA, USA), which were subsequently blocked for 0.5 hours with 3\% milk in Tris-buffered saline with Tween 20. The membranes were then probed with primary antibody diluted with $1 \%$ milk and incubated overnight at $4^{\circ} \mathrm{C}$. Signals were acquired with an enhanced chemiluminescence system after incubation with horseradish peroxidase-conjugated secondary antibodies (Jackson ImmunoResearch). Densitometric values were normalized versus $\beta$-actin.

\section{Statistical analyses}

Differences between groups were evaluated for statistical significance using one-way analysis of variance (ANOVA) with a Student's t-test. Null hypotheses of no difference were rejected if $P$ values were less than 0.05 .

\section{Results}

$A \beta 1-42$ reduced extracellular release of S100A9 in human THP-1 monocytes

To clarify the pathological mechanism related to S100A9 in $\mathrm{AD}$, we measured the extracellular release of S100A9 in response to stimulation with $A \beta 1-42$ in human THP-1 monocytes. We used A $\beta 1-42$ monomers instead of oligomers. The treatment of THP- 1 cells with A $\beta 1-42$ monomers significantly reduced the release of S100A9 at 24 hours in the conditioned media of THP-1 cells. This A 1 42-mediated decrease of S100A9 secretion occurred in a dose-dependent manner and maximal reduction of S100A9 secretion was found to occur at a concentration of $10 \mu \mathrm{M}$ A $31-42$ (Figure 1A, B). Notably, S100A9 secretion was consistently reduced when de novo mRNA expression and protein synthesis were inhibited by actinomycin D and cycloheximide, respectively. Thus, our data confirmed that A $\beta 1-42$ elicited a marked decrease of the extracellular S100A9 release in a dosedependent manner in human THP-1 monocytes, and that reduction of S100A9 release is dependent on both transcriptional and translational activities (Figure 2A,B).

\section{Intracellular $\mathrm{Ca}^{2+}$ level is involved in A 1 1-42-induced depletion of extracellular S100A9}

The increase of $\left[\mathrm{Ca}^{2+}\right]_{\mathrm{i}}$ may initiate the inflammatory response in activated microglia [27]. We observed that 10 $\mu \mathrm{M} A \beta$ oligomers extensively increased the level of $\left[\mathrm{Ca}^{2+}\right]_{i}$ in murine microglial BV2 cells as evaluated using the Fluo-3 AM method [18]. Thus, we investigated the role of intracellular $\mathrm{Ca}^{2+}$ levels in $\mathrm{A} \beta 1-42$-mediated reduction of S100A9 release and found that intracellular $\mathrm{Ca}^{2+}$ level is involved in human monocytic cells. We also observed that $10 \mu \mathrm{M}$ A $\beta 1-42$ monomers significantly increased intracellular $\mathrm{Ca}^{2+}$ levels in THP-1 cells as measured by Fluo-3 AM (Figure 3A,B). Furthermore, treatment of THP-1 cells with the $\mathrm{Ca}^{2+}$ ionophore, ionomycin, which induces $\left[\mathrm{Ca}^{2+}\right]_{\mathrm{i}}$

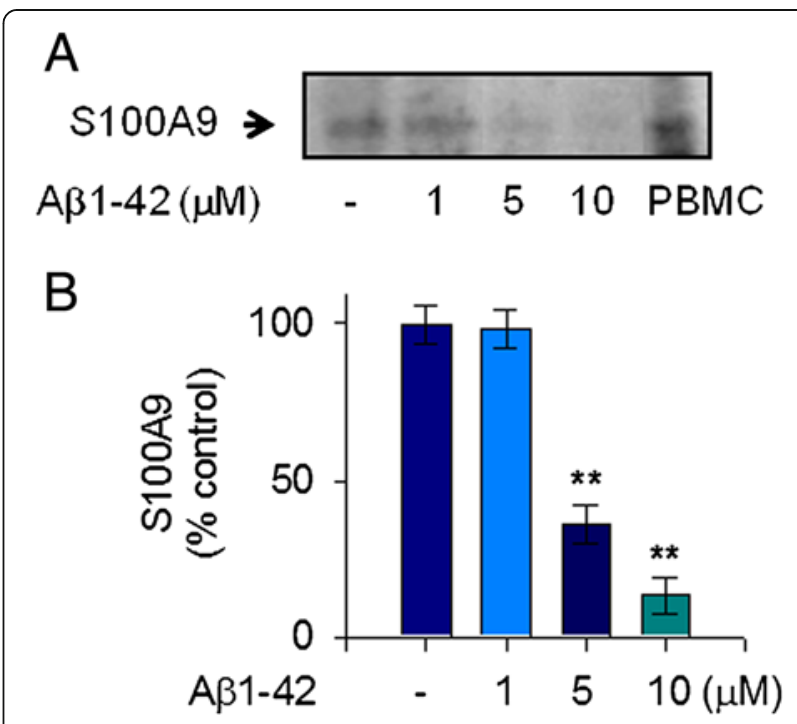

Figure 1 S100A9 release in response to $A \beta 1-42$ monomers in human monocytic THP-1 cells. To measure the extracellular release of S100A9 in response to mostly monomeric AB1-42 stimulation, THP-1 cells were incubated with either vehicle only (-) or increasing amounts of $A \beta 1-42$ for 24 hours in serum-free RPMI1640 medium supplemented with glucose (0.5\%). (A) The cell-free conditioned media were examined for S100A9 via protein immunoblot. Positive control for S100A9 was shown in human PBMC whole cell lysate. AB1-42 decreased S100A9 release in conditioned media in a dose-dependent manner. Results are representative of three independent experiments. (B) Densitometric quantification of analyses of (A), showing the levels of S100A9 release. All data are presented as the means $\pm \operatorname{SEM}(n=3)$. ${ }^{*} P<0.01$, versus vehicle treated samples. PBMC, peripheral blood mononuclear cells. 


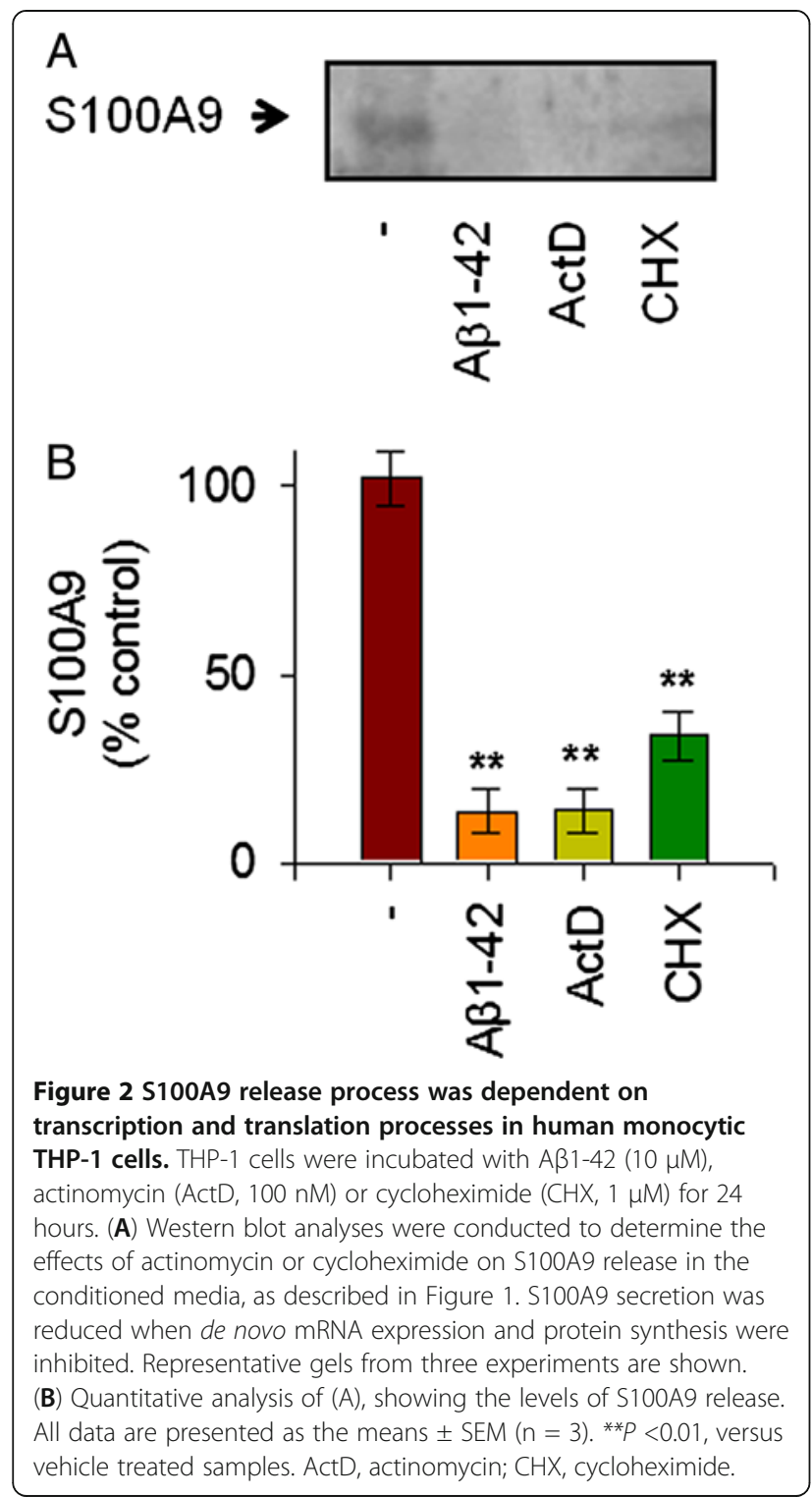

elevating intracellular $\mathrm{Ca}^{2+}$ concentration, induced the A $31-42$-evoked response decreasing the release of S100A9 (Figure 4A,D). Moreover, thapsigargin, an endoplasmic reticulum $\mathrm{Ca}^{2+}$ pump inhibitor, which induces an increase of intracellular $\mathrm{Ca}^{2+}$ level, also mimicked the $\mathrm{A} \beta 1-42$ -evoked effects. Concomitantly, the intracellular levels of S100A9 were increased in THP-1 cells treated with either ionomycin or thapsigargin as observed in $A \beta 1-42$ treated cells (Figure 4B,E). However, the A $\beta 1$-42-evoked response was significantly attenuated by either depletion of extracellular $\mathrm{Ca}^{2+}$ with EGTA or chelation of intracellular $\mathrm{Ca}^{2+}$ by BAPTA (Figure 4C,F). Together, these findings suggest that extracellular depletion of S100A9 in response to A $31-42$ monomers is dependent on an increase of intracellular $\mathrm{Ca}^{2+}$ and S100A9 levels in human THP-1 monocytes.

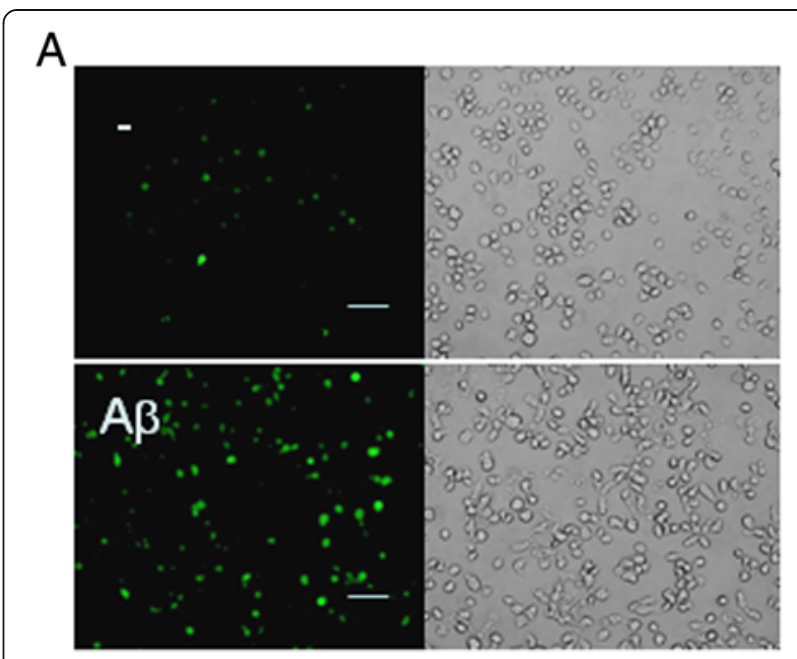

B

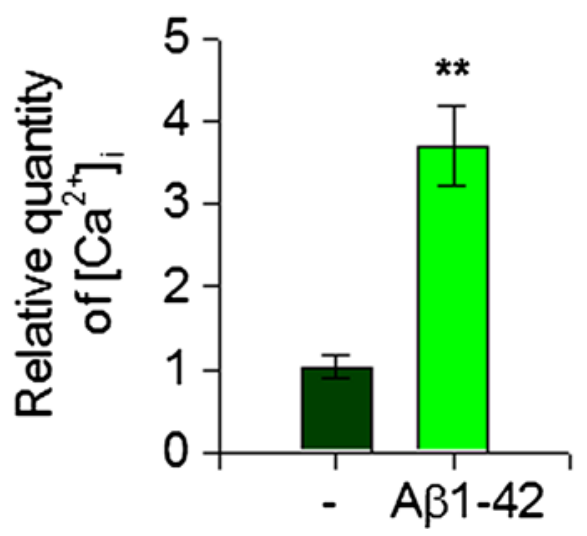

Figure $3 \mathrm{~A} \beta 1-42$ monomers increased $\left[\mathrm{Ca}^{2+}\right]_{i}$ in human monocytic THP-1 cells. (A) [Ca2+] images obtained by Fluo-3 AM at 24 hours after treatment with either vehicle only (-) or $10 \mu \mathrm{M}$ A 1-42 monomers (AB) in THP-1 cells. Scale bars represent $50 \mu \mathrm{m}$. (B) The histogram showing the ratio of $[\mathrm{Ca} 2+]_{\mathrm{i}}$ levels to vehicle treated group. All data are presented as the means $\pm \operatorname{SEM}(n=3)$. ${ }^{*} P<0.01$, versus vehicle treated samples. $A \beta$, amyloid beta.

\section{$A \beta 1-42$-induced depletion of extracellular S100A9 was} not associated with $A \beta 1-42-d e p e n d e n t$ cytotoxicity

To further describe the pathological mechanism related to $\mathrm{S} 100 \mathrm{~A} 9$ in $\mathrm{AD}$, the role of extracellular S100A9 depletion related to the $A \beta 1-42$-induced cytotoxicity was investigated. As shown in Figure 5, A $\beta 1-42$ treatment significantly increased cytotoxicity as measured by MTT reduction assay. Addition of rS100A9 protein into the cell culture supernatant did not significantly attenuate the A $31-42$-induced cytotoxicity (Figure 5A). Treatment with rS100A9 alone in the absence of A $\beta 1-42$ at concentrations up to $10 \mu \mathrm{g} / \mathrm{ml}$ had little effect on the cell viability and, as expected, a similar effect was observed with rS100A9 $9_{\text {hi }}$ (Figure 5B). In addition, depletion of S100A9 with siRNA did not significantly evoke cell toxicity (Figure 5C). These results demonstrate that extracellular depletion of S100A9 was not directly associated with the 


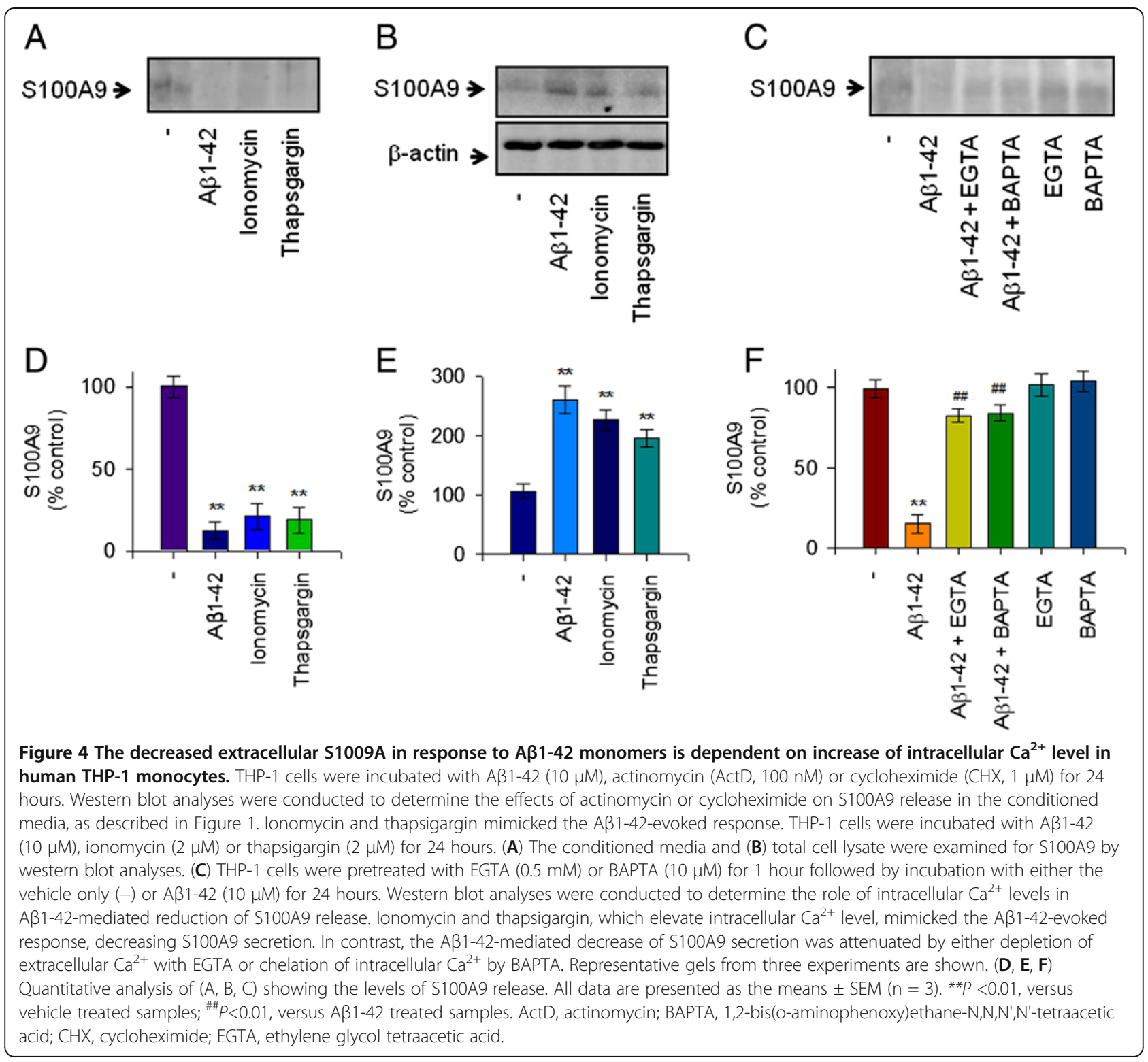

cytotoxicity in response to mostly $A \beta 1-42$ monomers in human monocytic THP-1 cells.

\section{$A \beta 1-42-i n d u c e d$ extracellular S100A9 depletion resulted in decreased antimicrobial activity}

Recent reports suggested that S100A9 acts as an additional antimicrobial peptide in the innate immune system, which provides immediate protection for the host against microbial challenge by recognizing the presence of microorganisms and preventing their tissue invasion, thus limiting microbial proliferation and inflammation [11,12]. We further investigated the antimicrobial activity of S100A9, which was released into the cell culture supernatants of THP-1 monocytes. The antimicrobial activities against
$E$ coli were assessed with the cell culture supernatants from vehicle or A $\beta-42$ treated THP-1 cells. Vehicle treated supernatant, which contained a significant amount of S100A9, demonstrated antimicrobial activity against $E$ coli. However, microbial growth was not decreased by the supernatant from $A \beta 1-42$ treated THP-1 cells in which the S100A9 level was significantly reduced (Figure 6A). Moreover, rS100A9 protein clearly elicited the antimicrobial peptide activity in vitro (Figure $6 \mathrm{~B}$ ), whereas $\mathrm{rS} 100 \mathrm{~A} 9_{\mathrm{hi}}$ had little activity. Consistently, immunodepletion of S100A9 with antiS100A9 antibodies blocked antimicrobial activity of the vehicle treated supernatant (Figure $6 \mathrm{C}$ ), confirming that the antimicrobial activity in the vehicle treated supernatant is S100A9-specific. 

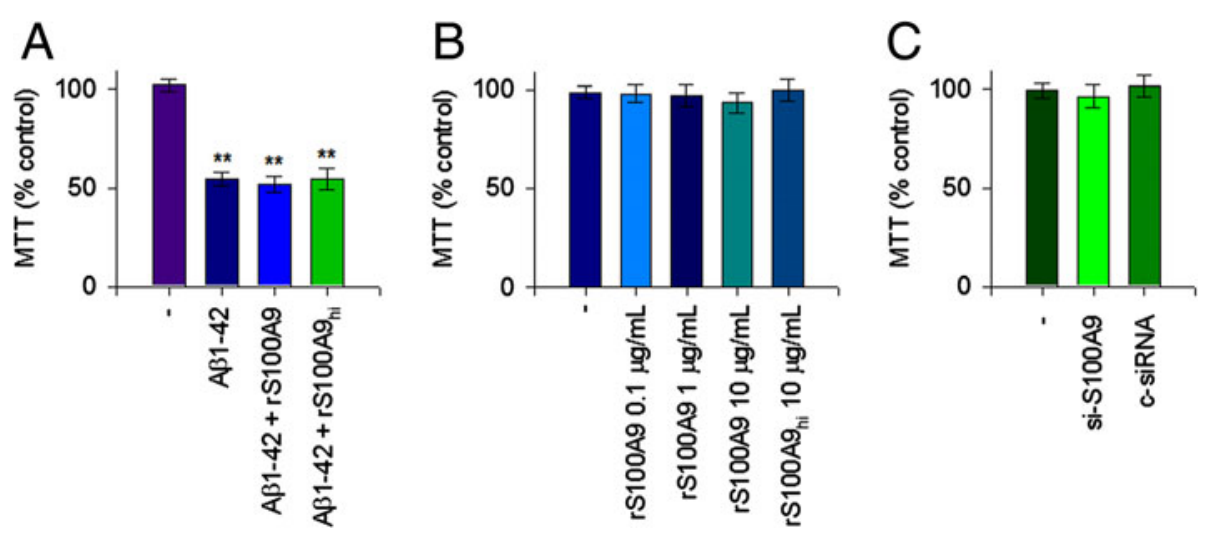

Figure 5 Extracellular S100A9 depletion by $A \beta 1-42$ monomers was not associated with $A \beta 1-42$-induced cytotoxicity. (A) To investigate the role of extracellular S100A9 depletion related to the AB1-42-induced cytotoxicity, THP-1 cells were incubated for 24 hours with AB1-42 $(10 \mu \mathrm{M})$ in the presence of $10 \mu \mathrm{g} / \mathrm{ml}$ recombinant S100A9 (rS100A9) or heat inactivated rS100A9 (rS100A9 hi . (B) THP-1 cells were also incubated with increasing amounts of rS100A9 alone as indicated for 24 hours. (C) THP-1 cells were transfected with S100A9 siRNA (100 ng/ml) or control siRNA (100 $\mathrm{ng} / \mathrm{ml}$ ) for 24 hours. The cell viability was measured by MTT reduction activity. rS100A9 protein did not attenuate the Aß1-42-induced cytotoxicity. rS100A9 alone in the absence of A $1-42$ also had little effect on the cell viability. Extracellular depletion of S100A9 with siRNA did not induce the cytotoxicity. Values are expressed as the means \pm SEM of triplicate experiments. MTT, 3(4,5-dimethylthiazol-2-yl)-2,5-diphenyltetrazolium bromide.

\section{Discussion}

The present study has four main findings concerning a mechanistic link between S100A9 and AD pathology. First, the mostly monomeric form of A $\beta 1-42$ markedly decreased S100A9 release into the cell culture supernatant of human THP-1 monocytes in parallel with increased intracellular S100A9. Second, this reduction of S100A9 release was accompanied by increased intracellular $\mathrm{Ca}^{2+}$ level.
Third, depletion of extracellular S100A9 in response to A $\beta 1-42$ monomers was not associated with $A \beta 1-42-$ induced cytotoxicity. Finally, A $\beta 1-42$-induced extracellular S100A9 depletion decreased antimicrobial activity of the culture supernatant from human monocytes, which was pathogenically challenged with A $\beta 1-42$. Our findings suggest that mostly $A \beta 1-42$ monomers negatively regulates the innate immune system by down-regulating the
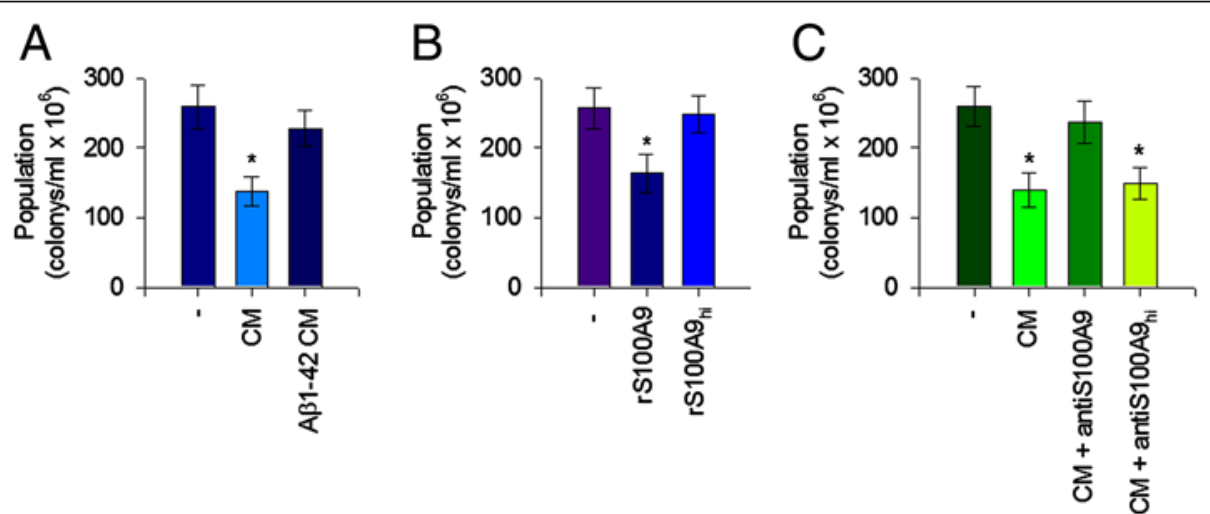

Figure 6 A $\beta 1-42$-induced extracellular S100A9 depletion resulted in decreased antimicrobial peptide activity. The antimicrobial activities against $E$ coli were assessed with the supernatants from vehicle or A $3-42$ treated THP-1 cells. (A) $E$ coli were cultured with serum-free RPMI-1640 media alone or the conditioned media from THP-1 cells treated with the vehicle or AB1-42 (10 $\mu \mathrm{M})$ for 24 hours. (B) To measure the antimicrobial activities of rS100A9 protein, E coli was cultured with rS100A9 or hi-rS100A9 (each $10 \mu \mathrm{g} / \mathrm{ml})$. (C) To inactivate antimicrobial activity of S100A9, which was released into the supernatants, the conditioned media from THP-1 cells treated with the vehicle for 24 hours were pretreated with anti-S100A9 antibodies or heat inactivated anti-S100A9 antibodies for 2 hours at $37^{\circ} \mathrm{C}$. E coli were then cultured with serum-free RPMI-1640 media alone or the conditioned media treated with anti-S100A9 antibodies or heat inactivated anti-S100A9 antibodies (anti-S100A9hi) as indicated. Data showed that vehicle treated supernatant, which contained a significant amount of S100A9, demonstrated antimicrobial activity against $E$ coli. Moreover, rS100A9 protein clearly elicited the antimicrobial peptide activity in vitro. Immunodepletion of S100A9 blocked antimicrobial activity of the vehicle treated supernatant. All data are presented as the means $\pm \operatorname{SEM}(n=3)$. ${ }^{*} P<0.01$, versus vehicle treated samples. $C M$, conditioned media. 
secretion of S100A9, which is likely a main mediator of the antimicrobial activity in the culture supernatants of human THP-1 monocytes.

S100A8, S100A9 and S100A12, as endogenous proteins associated with inflammation, are proposed to act as damage-associated molecular pattern (DAMP) initiators of innate immunity [28]. They are found at high concentrations in inflamed tissue, where neutrophils and monocytes are the most abundant cell types, and are released following neutrophil necrosis [29]. S100A8/S100A9 secretion may occur during interaction of phagocytes with endothelial cells and/or stimulation by lipopolysaccharide; IL-1 $\beta$ and TNF can promote S100A8/S100A9 release from monocytes [30,31]. Secretion may involve an energydependent process requiring protein kinase $\mathrm{C}$ activation in combination with a second calcium-dependent signal and interactions with microtubules [31,32]. Consistent with previous results that activated murine macrophages and human monocytes secreted significant amounts of S100A8 [33,34], this study has shown that human THP-1 monocytes secreted significant amounts of S100A9, which might be involved in autocrine/paracrine activities underlining the inflammatory process; although underlying molecular mechanisms of S100A9 secretion in human THP-1 monocytes remains to be determined.

S100A9 was increased within neuritic plaques and reactive glia, and was proposed to participate in the neuroinflammation associated with the pathogenesis of AD [17]. A recent study also reported that S100A9 interacts with $A \beta 1-40$ and induces its fibrillization, further supporting its association with $\mathrm{AD}$ [20]. Consistent with previous observations, our recent study has shown that S100A9 expression was increased in the brains of $\operatorname{Tg} 2576$ mice and AD patients [18]. The toxic oligomeric forms of $A \beta$ increased intracellular S100A9 levels in parallel with increases of $\left[\mathrm{Ca}^{2+}\right]_{i}$ and up-regulated S100A9 was found to be involved in the production of proinflammatory cytokines in BV2 cells [18]. Together, these findings propose the potential role of excessive S100A9 expression elicited by $A \beta$ oligomers in the neuroinflammation related to the learning and memory impairment in $\mathrm{AD}$ patients, and suggest S100A9 as a possible target for the pathogenesis of $\mathrm{AD}[18,19]$. On the other hand, it is noteworthy that the present study has shown for the first time, to our knowledge, that the mostly monomeric form of A $\beta 1-42$ led to a marked decrease of S100A9 secretion, accompanied by a mild increase of intracellular $\mathrm{Ca}^{2+}$ level in human THP-1 monocytes. Furthermore, since S100A9 has $\mathrm{Ca}^{2+}$ binding capacity, this extracellular depletion of S100A9 in response to A 1 1-42 monomers appears to be a consequence of increased intracellular S100A9 in parallel with the increased $\left[\mathrm{Ca}^{2+}\right]_{\mathrm{i}}$. A recent study has demonstrated a link between extracellular $\mathrm{Ca}^{2+}$ entry and a formation of
$\mathrm{Ca}^{2+}$-dependent heterocomplexes of S100A9, which is a probable prerequisite for its intracellular biological activities such as nicotinamide adenine dinucleotide phosphate-oxidase (NADPH oxidase) activation in myeloid cells [35]. This association of increased $\mathrm{Ca}^{2+}$ level with increased intracellular heterotetramers of S100A9 strongly supports our study.

The oligomeric forms of $A \beta$ exhibit stronger cytotoxicity than the monomeric form or the less toxic insoluble fibrillary form through their ability to bind lipid bilayers and cause uncontrolled influx of extracellular $\mathrm{Ca}^{2+}$, with devastating consequences for cellular $\mathrm{Ca}^{2+}$ homeostasis [36-38]. The present study, in which mostly A $1-42$ monomers instead of oligomers were used, has demonstrated that A $\beta 1-42$ monomers as measured by MTT assay exhibited cell toxicity in human THP-1 cells. Importantly, depletion of extracellular S100A9 release by A 1 1-42 monomers or siRNA was found to have little effect on the cell viability of human monocytic cells. Moreover, the recombinant S100A9 did not significantly evoke cell toxicity and had little effect on A $\beta 1-42$-induced cytotoxicity in human THP-1 monocytes.

While some aspects of excessive S100A9 could drive disease progression through the inflammation-induced up-regulation of proinflammatory cytokines, as shown in our previous study [18], there is also evidence that S100A9 may exert neuroprotective action. According to published reports, the proinflammatory functions of S100A9 tended to underplay important regulatory, antioxidant and protective properties $[9,10]$. S100A9 interaction with $\mathrm{A} \beta 1-40$ resulted in reduced S100A9 cytotoxicity by the binding of S100A9 toxic species to A $\beta 1-40$ amyloid structures [20]. Consequently, it was implied that secreted S100A9 during inflammation promoted the formation of amyloid plaques and that plaque formation may be the result of a protective response within the brain of $\mathrm{AD}$ patients, in part mediated by S100A9 [20]. Taken together, these findings suggest that S100A9 could mediate proinflammatory and antiinflammatory effects, depending on the monomeric or oligomeric forms of $A \beta$ species [39], the precise protocol used, including the excess or depleted concentrations, duration of exposure, overall immune environment, different cell types and species studied, and disease states; although the reason why S100A9 apparently mediated different effects on cell toxicity is not yet understood. Further studies are needed to clarify the apparent controversy, and to determine both intracellular and extracellular S100A9 using the toxic oligomeric form of $A \beta 1-42$.

Antimicrobial peptides may serve as a line of defense, and defensins are a family of antimicrobial peptides [40]. A previous report suggested that S100A9 (MRP14) is an additional antimicrobial peptide that forms calprotectin (MRP8/14) heterodimer with S100A8 (MRP8) [41]. 
Consequently, acting as an antimicrobial peptide in the innate immune system, S100A9 could provide immediate protection for the host against microbial challenge by recognizing the presence of microorganisms and preventing their tissue invasion, thus limiting microbial proliferation and inflammation. It is noteworthy that S100A9 is released more from damaged cells and may play a major antimicrobial role [42]. Importantly, our results have shown that $A \beta 1-42$-induced extracellular S100A9 depletion resulted in decreased antimicrobial activity of the culture supernatant of human THP-1 monocytes. This observation was confirmed by immunodepletion of S100A9 with anti-S100A9, which decreased the antimicrobial activity of the culture supernatant of the vehicle treated cells. Furthermore, the recombinant S100A9 elicited the antimicrobial peptide activity in vitro.

This is the first report to demonstrate that the mostly monomeric soluble form of $\mathrm{A} \beta 1-42$ negatively regulates the innate immune system by down-regulating the secretion of S100A9, which subsequently reduces the S100A9-dependent antimicrobial peptide activity in the culture supernatants of human THP-1 monocytes. This finding stands in stark contrast to recent reports demonstrating that $A \beta 1-42$ possess antimicrobial activity to kill bacteria under the appropriate conditions, which favor the formation of oligomers of $\mathrm{A} \beta$ peptide $[25,43]$. Further research will be required to demonstrate whether the oligomeric form of $A \beta 1-42$ would act together or in parallel with S100A9 to exert its antimicrobial property, and how different forms of $A \beta$ species such as the toxic oligomeric form of $A \beta 1-42$ versus the less toxic monomeric form of $\mathrm{A} \beta 1-42$ dysregulate or play a host defense role in vivo.

A large body of data supports a central role for neuroinflammation in $\mathrm{AD}$ neuropathology and $\mathrm{A} \beta$ as the source of $\mathrm{AD}$-associated inflammation $[4,6]$. Given that inflammatory response in the immunologically privileged CNS is mediated by the innate immune system, our data raise the possibility that rather than $A \beta$ acting as a sole independent initiator of neuroinflammation, increased $A \beta$ may trigger dysregulation of the innate immune system through depletion of extracellular S100A9 release from monocytes and decrease of its antimicrobial activity to protect against invading microbes. Increased microbial infection may further trigger a self-perpetuating innate immune response leading to an inappropriate inflammatory response in the CNS and subsequent production of $A \beta$, although the underlying cause of the aberrant neuroinflammation in $\mathrm{AD}$ patients still remains unclear. $\mathrm{A}$ number of studies reporting infection of the CNS of AD patients with various microbial pathogens [44-48] strongly support our study.

\section{Conclusion}

Collectively, our data indicate that A $\beta 1-42$ monomers decrease the secretion of S100A9 in situations where A $\beta 1-42$ enhances cytotoxicity. Furthermore, our findings suggest that the mostly monomeric form of $A \beta 1-42$ negatively regulates the innate immune system by downregulating the extracellular release of S100A9, which possesses antimicrobial peptide activity in human monocytes. The results of this study, at least in part, support the notion that increased amounts of $A \beta 1-42$ are not only toxic to human monocyte but also disrupt its normal physiological role for a host defense in the innate immune system, thereby contributing to an increased microbial infection in AD patients. Consequently, the results of this study have important implications for ongoing and future $\mathrm{AD}$ treatment strategies. However, the relevance of these findings in vivo remains to be clearly elucidated.

\section{Abbreviations}

ActD: Actinomycin; AD: Alzheimer's disease; ANOVA: Analysis of variance; AB: Amyloid beta; BAPTA: 1,2-bis(o-aminophenoxy)ethane-N,N,N',N'tetraacetic acid; CFU: Colony forming unit; CHX: Cycloheximide; CNS: Central nervous system; DAMP: Damage-associated molecular pattern; EGTA: Ethylene glycol tetraacetic acid; MTT: 3(4,5-dimethylthiazol-2-yl)-2, 5-diphenyltetrazolium bromide; NADPH oxidase: Nicotinamide adenine dinucleotide phosphate-oxidase; PBMC: Peripheral blood mononuclear cells; rS100A9: Recombinant S100A9; rS100A9 hi: Heat-inactivated rS100A9; SDS-PAGE: Sodium dodecyl sulfate polyacrylamide gel electrophoresis; siRNA: Small interfering RNA.

\section{Competing interests}

The authors declare that they have no competing interests.

\section{Authors' contributions}

The work presented here was carried out in collaboration between all authors. EL and JY carried out most of the laboratory experiments, analyzed the data and interpreted the results. $\mathrm{KC}$ helped in preparation of the manuscript. YS and YC conceived the idea for the study, and helped in designing methods and experiments. YC critically supervised the complete study. All the authors read and approved the final revised manuscript.

\section{Acknowledgements}

We are grateful to Dr Tessier at Laval University Hospital Center for providing human rS100A9 protein. This research was supported by Mid-Career Researcher Program through National Research Foundation grant funded by the Ministry of Education, Science and Technology (MEST) (2009-0086201), and by Basic Science Research Program through the National Research Foundation of Korea funded by the MEST (2010-0022658).

\section{Author details}

${ }^{1}$ Department of Microbiology, School of Medicine, Ewha Medical Research Institute, Ewha Womans University, 911-1, Mok-6-dong, Yangcheonku, Seoul 158-710, Republic of Korea. ${ }^{2}$ Department of Pharmacology, College of Medicine, Seoul National University, Seoul, Republic of Korea. ${ }^{3}$ Department of Pharmacology, College of Medicine, Gachon University, Incheon, Republic of Korea.

Received: 28 January 2013 Accepted: 24 April 2013

Published: 30 May 2013

\section{References}

1. Hampel H, Bürger K, Teipel SJ, Bokde AL, Zetterberg H, Blennow K: Core candidate neurochemical and imaging biomarkers of Alzheimer's disease. Alzheimers Dement 2008, 4:38-48. 
2. Chételat $G$, Villemagne $V L$, Pike $K E$, Ellis KA, Bourgeat $P$, Jones $G$, O'Keefe GJ, Salvado O, Szoeke C, Martins RN, Ames D, Masters CL, Rowe CC, Australian Imaging Biomarkers and Lifestyle Study of ageing (AIBL) Research Group: Independent contribution of temporal beta-amyloid deposition to memory decline in the pre-dementia phase of Alzheimer's disease. Brain 2011, 134:798-807.

3. Lukiw WJ: Amyloid beta $(A \beta)$ peptide modulators and other current treatment strategies for Alzheimer's disease (AD). Expert Opin Emerg Drugs 2012, 17:43-60.

4. Eikelenboom $\mathrm{P}$, Veerhuis $\mathrm{R}$, van Exel $\mathrm{E}$, Hoozemans JJ, Rozemuller AJ, van Gool WA: The early involvement of the innate immunity in the pathogenesis of late-onset Alzheimer's disease: neuropathological, epidemiological and genetic evidence. Curr Alzheimer Res 2011, 8:142-150.

5. Cribbs DH, Berchtold NC, Perreau V, Coleman PD, Rogers J, Tenner AJ, Cotman CW: Extensive innate immune gene activation accompanies brain aging, increasing vulnerability to cognitive decline and neurodegeneration: a microarray study. J Neuroinflammation 2012, 9:179.

6. Butchart J, Holmes C: Systemic and central immunity in Alzheimer's disease: therapeutic implications. CNS Neurosci Ther 2012, 18:64-76.

7. Perera C, McNeil HP, Geczy CL: S100 Calgranulins in inflammatory arthritis. Immunol Cell Biol 2010, 88:41-49.

8. van Lent PL, Grevers L, Blom AB, Sloetjes A, Mort JS, Vogl T, Nacken W, van den Berg WB, Roth J: Myeloid-related proteins S100A8/S100A9 regulate joint inflammation and cartilage destruction during antigeninduced arthritis. Ann Rheum Dis 2008, 67:1750-1758.

9. Sroussi HY, Berline J, Palefsky JM: Oxidation of methionine 63 and 83 regulates the effect of S100A9 on the migration of neutrophils in vitro. J Leukoc Biol 2007, 81:818-824.

10. Sroussi $H Y, L u Y$, Villines $D$, Sun $Y$ : The down regulation of neutrophil oxidative metabolism by S100A8 and S100A9: implication of the protease-activated receptor-2. Mol Immunol 2012, 50:42-48.

11. Erez O, Romero R, Tarca AL, Chaiworapongsa T, Kim YM, Than NG, Vaisbuch E, Draghici S, Tromp G: Differential expression pattern of genes encoding for antimicrobial peptides in the fetal membranes of patients with spontaneous preterm labor and intact membranes and those with preterm prelabor rupture of the membranes. J Matern Fetal Neonatal Med 2009, 22:1103-1115.

12. Hiroshima $Y$, Bando M, Kataoka M, Inagaki $Y$, Herzberg MC, Ross KF, Hosoi K, Nagata T, Kido J: Regulation of antimicrobial peptide expression in human gingival keratinocytes by interleukin-1a. Arch Oral Biol 2011, 56:761-767.

13. Zimmer DB, Chaplin J, Baldwin A, Rast M: S100-mediated signal transduction in the nervous system and neurological diseases. Cell Mol Biol 2005, 51:201-214.

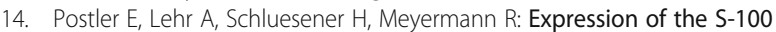
proteins MRP-8 and -14 in ischemic brain lesions. Glia 1997, 19:27-34.

15. Engel S, Schluesener H, Mittelbronn M, Seid K, Adjodah D, Wehner HD Meyermann R: Dynamics of microglial activation after human traumatic brain injury are revealed by delayed expression of macrophage-related proteins MRP8 and MRP14. Acta Neuropathol 2000, 100:313-322.

16. Heneka MT, Wiesinger $H$, Dumitrescu OL, Riederer $\mathrm{P}$, Feinstein DL, Klockgether $\mathrm{T}$ : Neuronal and glial coexpression of argininosuccinatesynthetase and inducible nitric oxide synthase in Alzheimer disease. J Neuropathol Exp Neurol 2001, 60:906-916.

17. Shepherd CE, Goyette J, Utter V, Rahimi F, Yang Z, Geczy CL, Halliday GM: Inflammatory S100A9 and S100A12 proteins in Alzheimer's disease. Neurobiol Aging 2006, 27:1554-1563.

18. Ha TY, Chang KA, Kim J, Kim HS, Kim S, Chong YH, Suh YH: S100a9 knockdown decreases the memory impairment and the neuropathology in Tg2576 mice. AD animal model. PLoS One 2010, 5:e8840.

19. Chang KA, Kim HJ, Suh YH: The role of S100a9 in the pathogenesis of Alzheimer's disease: the therapeutic effects of S100a9 knockdown or knockout. Neurodegener Dis 2012, 10:27-29.

20. Zhang C, Liu Y, Gilthorpe J, van der Maarel JR: MRP14 (S100A9) protein interacts with Alzheimer beta-amyloid peptide and induces its fibrillization. PLoS One 2012, 7:e32953.

21. Lee EO, Kang $\lrcorner$, Chong $Y H$ : The amyloid-beta peptide suppresses transforming growth factor-beta1-induced matrix metalloproteinase-2 production via Smad7 expression in human monocytic THP-1 cells. J Biol Chem 2005, 280:7845-7853.

22. Ryckman C, Vandal $K$, Rouleau $P$, Talbot $M$, Tessier PA: Proinflammatory activities of S100: proteins S100A8, S100A9, and S100A8/A9 induce neutrophil chemotaxis and adhesion. J Immunol 2003, 170:3233-3242.
23. Chong $Y H$, Sung $J H$, Shin $S A$, Chung $J H$, Suh $Y H$ : Effects of the betaamyloid and carboxyl-terminal fragment of Alzheimer's amyloid precursor protein on the production of the tumor necrosis factor-alpha and matrix metalloproteinase-9 by human monocytic THP-1. J Biol Chem 2001, 276:23511-23517.

24. Lee EO, Kim SE, Park HK, Kang JL, Chong YH: Extracellular HIV-1 Tat upregulates TNF- $a$ dependent MCP-1/CCL2 production via activation of ERK1/2 pathway in rat hippocampal slice cultures: inhibition by resveratrol, a polyphenolicphytostilbene. Exp Neurol 2011, 229:399-408.

25. Soscia SJ, Kirby JE, Washicosky KJ, Tucker SM, Ingelsson M, Hyman B, Burton MA, Goldstein LE, Duong S, Tanzi RE, Moir RD: The Alzheimer's diseaseassociated amyloid beta-protein is an antimicrobial peptide. PLoS One 2010, 5:e9505.

26. Chong YH, Shin YJ, Lee EO, Kayed R, Glabe CG, Tenner AJ: ERK1/2 activation mediates Abeta oligomer-induced neurotoxicity via caspase-3 activation and tau cleavage in rat organotypic hippocampal slice cultures. J Biol Chem 2006, 281:20315-20325.

27. Malm TM, Koistinaho M, Parepalo M, Vatanen T, Ooka A, Karlsson S, Koistinaho J: Bone-marrow-derived cells contribute to the recruitment of microglial cells in response to beta-amyloid deposition in APP/PS1 double transgenic Alzheimer mice. Neurobiol Dis 2005, 18:134-142.

28. Foell D, Wittkowski H, Vogl T, Roth J: S100 proteins expressed in phagocytes: a novel group of damage-associated molecular pattern molecules. J Leukoc Biol 2007, 81:28-37.

29. Voganatsi A, Panyutich A, Miyasaki KT, Murthy RK: Mechanism of extracellular release of human neutrophil calprotectin complex. J Leukoc Biol 2001, 70:130-134.

30. Kido J, Hayashi N, Kataoka M, Nagata T: Calprotectin expression in human monocytes:induction by porphyromonasgingivalis lipopolysaccharide tumor necrosis factor-alpha, and interleukin-1beta. J Periodontol 2005, 76:437-442.

31. Rammes A, Roth J, Goebeler M, Klempt M, Hartmann M, Sorg C: Myeloidrelated protein (MRP) 8 and MRP14, calcium-binding proteins of the S100 family, are secreted by activated monocytes via a novel, tubulindependent pathway. J Biol Chem 1997, 272:9496-9502.

32. Vogl T, Ludwig S, Goebeler M, Strey A, Thorey IS, Reichelt R, Foell D, Gerke V, Manitz MP, Nacken W, Werner S, Sorg C, Roth J: MRP8 and MRP14 control microtubule reorganization during transendothelial migration of phagocytes. Blood 2004, 104:4260-4268.

33. Xu K, Geczy CL: IFN-gamma and TNF regulate macrophage expression of the chemotactic S100 protein S100A8. J Immunol 2000, 164:4916-4923.

34. Xu K, Yen T, Geczy CL: IL-10 up-regulates macrophage expression of the S100 protein S100A8. J Immunol 2001, 166:6358-6366.

35. Bréchard S, Plançon S, Tschirhart EJ: New insights into the regulation of neutrophil NADPH oxidase activity in the phagosome: a focus on the role of lipid and $\mathrm{Ca}(2+)$ signaling. Antioxid Redox Signal 2013, 18:661-676.

36. Demuro A, Parker I, Stutzmann GE: Calcium signaling and amyloid toxicity in Alzheimer disease. J Biol Chem 2010, 285:12463-12468.

37. Demuro A, Smith $M$, Parker I: Single-channel $\mathrm{Ca}(2+)$ imaging implicates A 1-42 amyloid pores in Alzheimer's disease pathology. J Cell Biol 2011, 195:515-524.

38. Camandola S, Mattson MP: Aberrant subcellular neuronal calcium regulation in aging and Alzheimer's disease. Biochim Biophys Acta 2011, 1813:965-973

39. Ladiwala AR, Litt J, Kane RS, Aucoin DS, Smith SO, Ranjan S, Davis J, Van Nostrand WE, Tessier PM: Conformational differences between two amyloid $\beta$ oligomers of similar size and dissimilar toxicity. J Biol Chem 2012, 287:24765-24773.

40. Medzhitov R: Recognition of microorganisms and activation of the immune response. Nature 2007, 449:819-826.

41. Striz I, Trebichavsky I: Calprotectin - a pleiotropic molecule in acute and chronic inflammation. Physiol Res 2004, 53:245-253.

42. Swangchan-Uthai T, Lavender CR, Cheng Z, Fouladi-Nashta AA, Wathes DC: Time course of defense mechanisms in bovine endometrium in response to lipopolysaccharide. Biol Reprod 2012, 87:135.

43. Kagan $\mathrm{BL}$, Jang $\mathrm{H}$, Capone $\mathrm{R}$, TeranArce $\mathrm{F}$, Ramachandran $\mathrm{S}$, Lal R, Nussinov R: Antimicrobial properties of amyloid peptides. Mol Pharm 2012, 9:708-717.

44. Honjo K, van Reekum R, Verhoeff NP: Alzheimer's disease and infection: do infectious agents contribute to progression of Alzheimer's disease? Alzheimers Dement 2009, 5:348-360. 
45. Itzhaki RF, Wozniak MA, Appelt DM, Balin BJ: Infiltration of the brain by pathogens causes Alzheimer's disease. Neurobiol Aging 2004, 25:619-627

46. Miklossy J: Emerging roles of pathogens in Alzheimer disease. Expert Rev Mol Med 2011, 13:e30.

47. Youngsteadt E: Virology. Alzheimer's risk factor also aids HIV. Science 2008, 320:1577.

48. Miklossy J: Alzheimer's disease - a neurospirochetosis. Analysis of the evidence following Koch's and Hill's criteria. J Neuroinflammation 2011, 8:90.

doi:10.1186/1742-2094-10-68

Cite this article as: Lee et al: Amyloid- $\beta$ peptide-induced extracellular S100A9 depletion is associated with decrease of antimicrobial peptide activity in human THP-1 monocytes. Journal of Neuroinflammation 2013 10:68.

\section{Submit your next manuscript to BioMed Central and take full advantage of:}

- Convenient online submission

- Thorough peer review

- No space constraints or color figure charges

- Immediate publication on acceptance

- Inclusion in PubMed, CAS, Scopus and Google Scholar

- Research which is freely available for redistribution 